\title{
Pretreatment with Astragaloside IV protects human umbilical vein endothelial cells from hydrogen peroxide induced oxidative stress and cell dysfunction via inhibiting eNOS uncoupling and NADPH oxidase- ROS-NF-KB pathway
}

\begin{tabular}{|r|l|}
\hline Journal: & Canadian Journal of Physiology and Pharmacology \\
\hline Manuscript ID & cjpp-2015-0572.R1 \\
\hline Manuscript Type: & Article \\
\hline Date Submitted by the Author: & 05-Apr-2016 \\
\hline Complete List of Authors: & $\begin{array}{l}\text { Xu, Chonghua; Liaoning Medical University } \\
\text { Tang, Futian; Liaoning Medical University } \\
\text { Lu, Meili; 3. Key Laboratory of Cardiovascular and Cerebrovascular Drug } \\
\text { Research of Liaoning Province, } \\
\text { Yang, Jing; Liaoning Medical University } \\
\text { Han, Ronghui; Liaoning Medical University } \\
\text { Mei, Meng; Liaoning Medical University } \\
\text { Hu, Jin; Liaoning Medical University } \\
\text { Wang, Hongxin; Liaoning Medical University }\end{array}$ \\
\hline Keyword: & $\begin{array}{l}\text { Astragaloside IV, hydrogen peroxide, eNOS uncoupling, NF-KB, NADPH } \\
\text { oxidase }\end{array}$ \\
\hline &
\end{tabular}

\section{SCHOLARONE ${ }^{m}$


Pretreatment with Astragaloside IV protects human umbilical vein endothelial cells from hydrogen peroxide induced oxidative stress and cell dysfunction via inhibiting eNOS uncoupling and NADPH oxidase- ROS-NF-кB pathway

Xu, Chonghua ${ }^{\text {al }}$; Tang, Futian ${ }^{\text {a1 }}$, Lu, Meili ${ }^{\text {a }}$ Y Yang, Jing ${ }^{\text {a }}$; Han, Ronghui ${ }^{a}$; Mei, Meng ${ }^{\text {a }}$; Hu, Jin ${ }^{a}$ and Wang, Hongxin ${ }^{a^{*}}$

${ }^{\text {a }}$ Key Laboratory of Cardiovascular ands Cerebrovascular Drug Research of Liaoning Province, Liaoning Medical University, Jinzhou 121001, China

*Corresponding author: Hongxin Wang (Tel: +8613304169343. Email: hongxinwang@lnmu.edu.cn)

${ }^{1}$ These authors have contributed equally to this work 


\begin{abstract}
Endothelial cell injury caused by reactive oxygen species (ROS) plays a critical role in the pathogenesis of cardiovascular disorders. Astragaloside IV (AsIV) possesses potent antioxidant properties against oxidative stress through undefined mechanism(s). We sought to investigate whether AsIV protects human umbilical vein endothelial cells (HUVECs) from hydrogen peroxide $\left(\mathrm{H}_{2} \mathrm{O}_{2}\right)$ induced oxidative stress focusing on eNOS uncoupling and the NADPH oxidase- ROS-NF- $\kappa$ B pathway. Compared with HUVECs incubated with $\mathrm{H}_{2} \mathrm{O}_{2}$ alone, pretreatment with AsIV significantly increased the viability of HUVECs, which was accompanied with apparent increase in nitric oxide (NO) production and decrease in intracellular superoxide anion production. Furthermore, pretreatment with AsIV increased endothelial nitric oxide synthase (eNOS) dimer/monomer ratio and its critical cofactor tetrahydrobiopterin $\left(\mathrm{BH}_{4}\right)$ content, decreased Nox4 protein expression, the most abundant Nox isoform in HUVECs, inhibited translocation of NF- $\kappa \mathrm{B}$ p65 subunit into nuclear fraction while enhanced the

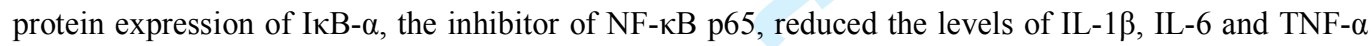
in HUVECs medium, and decreased iNOS protein expression,. These results suggest that AsIV may protect HUVECs from $\mathrm{H}_{2} \mathrm{O}_{2}$ induced oxidative stress via inhibiting NADPH oxidase-ROS-NF- $\kappa \mathrm{B}$ pathway and eNOS uncoupling.
\end{abstract}

\title{
Keywords
}

Astragaloside IV; human umbilical vein endothelial cells; hydrogen peroxide; eNOS uncoupling;

NF-кB; NADPH oxidase; tetrahydrobiopterin; superoxide anion 


\section{Introduction}

As is demonstrated that oxidative stress plays pivotal role in the development of the endothelial dysfunction associated with many cardiovascular disease (Hermida and Balligand 2014; Makarenko et al. 2014; Napoli et al. 2004; Sena et al. 2013). Evidence has shown that endothelium-dependent vascular function was damaged in ventricular hypertrophy in vivo, which was associated with enhanced oxidative stress ( Davel et al. 2006; Davel et al. 2014). Nitric oxide (NO), a key regulator of endothelial function, is generated under the catalysis of endothelial nitric oxide synthase (eNOS) (Forstermann 2010). Enhanced reactive oxygen species (ROS) production reduces the availability of NO by chemical inactivation to form toxic peroxynitrite (OONO-) which can uncouple endothelial NO synthase. The major source of ROS in the endothelial cells (ECs) is the nicotinamide adenine dinucleotide phosphate oxidase (NADPH oxidase) system (Wang et al. 2015), which consists isoforms of Nox1, Nox2, Nox4 and Nox5 (Vendrov et al. 2015). Nox4 plays the central role in producing ROS in ECs, thus the increased Nox4 expression is associated with early progression of cardiovascular disease (Zhao et al. 2015). Instead of producing NO, uncoupled eNOS becomes a dysfunctional superoxide-generating enzyme, which produces superoxide anion contributing to vascular oxidative stress (Forstermann and Munzel 2006; Forstermann 2010). In addition, $\mathrm{BH}_{4}$ is a crucial co-factor for normal NOS function, and eNOS becomes uncoupled for its depletion and/or oxidation (Gorren and Mayer 2002; Moens and Kass 2006). So protecting $\mathrm{BH}_{4}$ from oxidation is an effective way to ameliorate the injury of vascular endothelial cells. It has been demonstrated that $\mathrm{H}_{2} \mathrm{O}_{2}$ acts as a signaling molecule for both NADPH oxidases and eNOS, which play a critical role in maintaining the balance between superoxide anion $\left(\mathrm{O}^{2-}\right)$ and NO. Exposure of endothelial cells to $\mathrm{H}_{2} \mathrm{O}_{2}$ caused upregulation of eNOS after several hours (Drummond et al. 2000), and on a shorter time scale, 
activated eNOS through phosphorylation (Cai et al. 2003b). However, NO bioavailability is decreased when endothelial cells are continuously exposed to $\mathrm{H}_{2} \mathrm{O}_{2}$ for an intermediate time period (Jaimes et al. 2001). Study has shown that early $\mathrm{H}_{2} \mathrm{O}_{2}$-induced endothelial dysfunction was associated with a decreased $\mathrm{BH}_{4}$ level and increased superoxide anion production (Boulden et al. 2006).

Furthermore, ROS serve as the common intracellular messengers of nuclear factor-kappa B $(\mathrm{NF}-\kappa \mathrm{B})$ activation and plays a critical role in the development of inflammatory response (Olejarz et al. 2014). There is increasing evidence that redox-sensitive signaling pathways, such as NF- $\mathrm{B}$, are involved in oxidative stress-mediated endothelial cell dysfunction (Chen and Mehta 2004). Moreover, TLR4 triggers activation of MAPK/NF-kappa B signaling pathways to induce iNOS gene overexpression, which could aggravate inflammation (Kang et al. 2013).

Astragaloside IV (AsIV), which is used in traditional Chinese medicine, is extracted from the Chinese herb Astragalus membranaceus. Our previous studies confirmed that AsIV can suppressmyocardial hypertrophy and attenuate apoptosis of hypertrophic cardiomyocyte (Lu et al. 2015; Yang et al. 2013; Zhang et al. 2015), which may be partially due to its anti-oxidative, anti-inflammatory and anti-apoptotic activities that might be endothelium-protective under pathophysiological conditions. Previous study has been demonstrated that AsIV inhibits $\mathrm{H}_{2} \mathrm{O}_{2}$-induced HUVECs apoptosis by suppressing Nox4 expression(Ma et al. 2015). Previous study also has shown that the anti-inflammatory activity of AsIV is mediated by inhibition of NF-kappaB activation and adhesion molecule expression (Zhang et al. 2003). Based on these previous studies, the purpose of our present study is to investigate the protective effects of AsIV against damage of HUVECs induced by $\mathrm{H}_{2} \mathrm{O}_{2}$ focusing on eNOS uncoupling and the NADPH oxidase-ROS-NF- $\kappa$ B pathway. 


\section{Materials and Methods}

\section{Regent and antibodies}

Human umbilical vein endothelial cells (HUVECs) were obtained from ScienCell. Astragaloside IV (purity $>98 \%$ ) was purchased from CHENGDU CONBON BIO-TECH CO., LTD (Chengdu, China). Hydrogen peroxide (MW 247.72, CAS N5984-95-2), (6R)-5, 6, 7, 8-Tetrahydrobiopterin dihydrochloride (MW 34.01, CAS 7722-84-1) were purchased from Sigma (St, Louio, USA). Hydroethidine was purchased from AAT Bioquest. Antibodies against IкB- $\alpha$, p65, eNOS, Nox4, lamin B, GAPDH and $\beta$-actin were from abcam (Cambridge, MA, USA). The primers of GAPDH, Nox4, eNOS were obtained from TaKaRa Biotechnology Co. (Dalian, China). NO kits were from Nanjing Jiancheng Bioengineering Institute (Nanjing, China). Nuclear and cytoplasmic protein extraction kits were from Beyotime Biotechnology (Shanghai, China). Enzyme-linked immunosorbent assay (ELISA) kits for IL-1 $\beta$, IL-6 and TNF- $\alpha$ were from R\&D Systems (Minneapolis, MN, USA). DMEM was from Gibco-BRL Company (Gibco, USA).

\section{Cell culture protocol}

HUVECs were cultured in DMEM medium containing 10\% fetal bovine serum (FBS), 100 $\mathrm{U} / \mathrm{ml}$ penicillin and $100 \mu \mathrm{g} / \mathrm{ml}$ streptomycin in a humidified atmosphere of $5 \% \mathrm{CO}_{2}$ at $37^{\circ} \mathrm{C}$. Medium was changed every 48 hours until cells reached $80 \%$ to $90 \%$ confluency. At confluency, cells were either subcultured or used for experiments. $48 \mathrm{~h}$ after cells were incubated in $15 \%$ serum medium, the culture medium were replaced by a lower serum (1\%) DMEM to avoid serum components affect the results. The cells were randomly divided into five groups as follows: (1) the control (Con); (2) the model group: cells were treated with $\mathrm{H}_{2} \mathrm{O}_{2} 200 \mu \mathrm{mol} / \mathrm{l}$ for $24 \mathrm{~h}\left(\mathrm{H}_{2} \mathrm{O}_{2}\right)$; (3) $\mathrm{H}_{2} \mathrm{O}_{2}+\mathrm{AsIV}(25 \mu \mathrm{mol} / \mathrm{L})$ 
$\left(\right.$ AsIV L $\left.+\mathrm{H}_{2} \mathrm{O}_{2}\right)$; (4) $\mathrm{H}_{2} \mathrm{O}_{2}+\operatorname{AsIV}(50 \mu \mathrm{mol} / \mathrm{L})\left(\operatorname{AsIV} \mathrm{M}+\mathrm{H}_{2} \mathrm{O}_{2}\right) ;(5) \mathrm{H}_{2} \mathrm{O}_{2}+\operatorname{AsIV}(100 \mu \mathrm{mol} / \mathrm{L})$

(AsIV H+ $\mathrm{H}_{2} \mathrm{O}_{2}$ ); (6) AsIV $100 \mu \mathrm{mol} / \mathrm{L}$ (AsIV H). The cells were treated with AsIV in the medium for $1 \mathrm{~h}$ prior to exposure to $\mathrm{H}_{2} \mathrm{O}_{2}$.

\section{Determination of viability by MTT assay}

The MTT assay is based on the previous study (Jin et al. 2014). Briefly, cells were cultured at density of $5 \times 10^{4}$ cells $/ \mathrm{ml}$ in 96 -well plates, at the indicated time points after the corresponding treatment, the culture supernatant was removed, and the cells were washed with PBS, then incubated with MTT $(5 \mathrm{mg} / \mathrm{ml})$ in culture medium at $37{ }^{\circ} \mathrm{C}$ for $4 \mathrm{~h}$. After MTT removal, $100 \mu$ of DMSO was added to the wells to solubilize the crystals. The absorption values were measured at $490 \mathrm{~nm}$ using ELISA reader (Perlong Medical, Beijing, China). The viability of HUVECs in each well was presented as percentage of control cells.

\section{Measurement of nitrite/nitrate}

Oxidation products of NO (nitrite and nitrate) were assayed as a measure of total NO synthesis (Qiu et al. 2010). Nitric oxide in the medium of HUVECs was measured using a commercially available nitrite/nitrate assay kit according to manufacturer's instructions. The OD values were measured in 550nm by ultraviolet spectrophotometer (Mapada Instruments, Shanghai, China).

\section{Measurement of cellular superoxide anion production}

The intracellular superoxide anion in intact HUVECs was determined by dihydroethidium (DHE) that can be oxidized by superoxide anion. DHE can intercalate into nuclear DNA and yield fluorescent 
ethidium bromide, this dye has been shown to specifically detect superoxide anion in endothelial cells (Cai et al. 2005). Briefly, cells in 96-well plates were rinsed with phosphate-buffered saline and incubated with $10 \mu \mathrm{mol} / \mathrm{L}$ dihydroethidium in phosphate-buffered saline of $\mathrm{pH}$ 7.4, in a light-protected environment at $37{ }^{\circ} \mathrm{C}$. After $30 \mathrm{~min}$, fluorescent microscopic images were obtained using identical acquisition parameters (excitation at $488 \mathrm{~nm}$; emission at $610 \mathrm{~nm}$ ) (Leica, Germany). Then the cell fluorescent was detected by the Fluorescence microplate reader at 530nm (Bio Tek, winooski VT, USA).

\section{HPLC analysis for (6R)-5, 6, 7, 8-tetrahydrobiopterin $\left(\mathrm{BH}_{4}\right)$ content in cell lysates}

Measurements of biopterin levels in cell lysates was performed as described previously (Benson et al. 2013; Cai et al. 2005). Cell pellets from 12-well plates were lysed in cold extraction buffer (50 mmol/L Tris-HCl, 1mmol/L EDTA, 1 mmol/L dithiothreitol; $\mathrm{pH} 7.4$ ) and then divided equal volumes into two tubes containing alkaline $(1 \mathrm{~mol} / \mathrm{L} \mathrm{NaOH})$ or acid $\left(1 \mathrm{~mol} / \mathrm{L} \mathrm{H}_{3} \mathrm{PO}_{4}\right)$. A solution of $1 \%$ iodine in $2 \%$ potassium iodide was added to each tube. The total amount of biopterin obtained by oxidation in acid condition $\left(\mathrm{BH}_{4}+\mathrm{BH}_{2}+\mathrm{B}\right)$ and the content of $\mathrm{BH}_{2}$ and free biopterin $\left(\mathrm{BH}_{2}+\mathrm{B}\right)$ were determined in alkaline oxidation using high-performance liquid chromatography with fluorescence detection (350 nm excitation,450 $\mathrm{nm}$ emission)(SHIMADZU, Japan). Therefore, $\mathrm{BH}_{4}$ levels were obtained from difference between total $\left(\mathrm{BH}_{4}\right.$ plus $\mathrm{BH}_{2}$ plus biopterin) and alkaline-stable oxidized $\left(\mathrm{BH}_{2}\right.$ plus biopterin) biopterin. $\mathrm{BH}_{4}$ levels were normalized to protein concentration. Protein concentration was measured using the Bio-Rad protein assay.

\section{Measurement of IL-1ß, IL-6 and TNF- $\alpha$ levels by ELISA}


According to the instructions on the ELISA kit, the medium of HUVECs was used to determine the contents of IL-1 $\beta$, IL- 6 and TNF- $\alpha$. The optical density (OD) value of each well was measured at a wavelength of $450 \mathrm{~nm}$. A standard curve was constructed and the curve equation was calculated. By substituting the OD values of the samples into the equation of the standard curve, IL-1 $\beta$, IL- 6 and TNF- $\alpha$ levels were calculated.

\section{Western-blot analysis for eNOS, iNOS, Nox4, p65 and IкB- $\alpha$}

Total protein extraction Cells were lysed in Radio Immunoprecipitation Assay (RIPA) Lysis Buffer $(50 \mathrm{mmol} / 1$ Tris-HCl, $137 \mathrm{mmol} / 1 \mathrm{NaCl}, 10 \%$ glycerol, $100 \mu \mathrm{mol} / 1$ sodium orthovanadate, $1 \mathrm{mmol} / 1$ phenylmethylsulfonylfluoride, $10 \mu \mathrm{g} / \mathrm{ml}$ aprotinin, $10 \mu \mathrm{g} / \mathrm{ml}$ leupeptin, and $1 \%$ Nonident P-40; $\mathrm{pH}$ 7.4) on ice. The lysate was centrifuged at $12,000 \mathrm{~g}$ at $4 \square$ for $15 \mathrm{~min}$ and the supernatant were taken into another tubes and stored at $-80^{\circ} \mathrm{C}$.

Nuclear-cytosolic protein extraction Cytoplasmic and nuclear protein extracts were prepared from HUVECs using the nuclear and cytoplasmic protein extraction kit according to the manufacturer's instructions.

The protein concentrations were measured by BCA kit. After being boiled, protein samples $(30 \mu \mathrm{g})$ were subjected to SDS-PAGE in $10 \%$ polyacrylamide gels, respectively for Nox4, nuclear-cytosolic p65, cytosolic I $\mathrm{KB}-\alpha, 6 \%$ polyacrylamide gels for eNOS total protein and iNOS protein expressionFor the eNOS dimmer/monomer analysis, non-boiled protein samples $(30 \mu \mathrm{g})$ were used in low temperature for SDS-PAGE by $6 \%$ polyacrylamide.

\section{RT-PCR for eNOS and Nox4 mRNA measurement}


After treatment, total RNA was isolated from HUVECs with Trizol reagent following the manufacture's instruction. Then 500ng RNA was digested with DNase and reverse transcribed to cDNA using AMV reverse transcriptase. Afterwards cDNA was amplified by PCR with primers. For GAPDH (217 bp): forward primer, TCC CTG AGC TGA ACG GGA $A G$; reverse primer, GGA GGA GTG GGT GTC GCT GCT GT. For eNOS (139bp): forward primer, TTT GCC CTT ATG GAT GTG $A A G$; reverse primer, $C G C A T C A A A G A A A G C T C A G T C$. For Nox4 (129bp): forward primer, $C A G$ AAG GTT CCA AGC AGG AG; reverse primer, GTT GAG GGC ATT CAC CAG AT. The target products were assessed by $1.5 \%$ agarose gel with GoldView. GAPDH were used as control for normalization.

\section{Statistical analysis}

Results are expressed as means $\pm \mathrm{SE}$, and $\mathrm{n}$ represents the number of independent experiments. Statistical analysis was analyzed by one-way ANOVA followed by the Bonferroni post hoc test. A value of $P<0.05$ was considered significant.

\section{Results}

\section{Effects of AsIV on the viability of HUVECs}

As shown in Fig.1, after exposure to $200 \mu \mathrm{M}$ of $\mathrm{H}_{2} \mathrm{O}_{2}$, the viability of HUVECs decreased markedly compared with control. However, pre-incubation of HUVECs with different concentrations of $\operatorname{AsIV}(20,50,100 \mu \mathrm{M})$ significantly increased the viability of $\mathrm{H}_{2} \mathrm{O}_{2}$-treated HUVECs. Otherwise, compared with the control group, treatment with AsIV alone could also increase the viability HUVECs. 


\section{AsIV increased NO levels in $\mathrm{H}_{2} \mathrm{O}_{2}$-treated HUVECs}

As shown in Fig.2, exposure to $\mathrm{H}_{2} \mathrm{O}_{2}$ for $24 \mathrm{~h}$ markedly decreased the NO (nitrite and nitrate) levels in the medium, while pretreatment of HUVECs with different concentrations of AsIV inhibited the decrease in $\mathrm{NO}$ content of $\mathrm{H}_{2} \mathrm{O}_{2}$-treated cells in a concentration-dependent manner. Compared with the control group, treatment with AsIV alone increased the NO concentration of the medium. This suggested that AsIV may ameliorate $\mathrm{H}_{2} \mathrm{O}_{2}$-induced cell dysfunction by enhancing the bioavailability of NO.

\section{AsIV inhibited $\mathrm{H}_{2} \mathrm{O}_{2}$-induced superoxide anion generation in HUVECs}

There was an increase in superoxide anion production of HUVECs treated with $\mathrm{H}_{2} \mathrm{O}_{2}$ for 24 hours as shown in DHE fluorescence images (Fig.3a) and the OD value at 530nm (Fig.3b), while pretreatment with AsIV markedly inhibited the increased in superoxide anion production. The results suggested that AsIV could suppress superoxide anion generation induced by $\mathrm{H}_{2} \mathrm{O}_{2}$. There were no difference between the control group and the AsIV $\mathrm{H}$ group.

\section{AsIV increased $\mathrm{BH}_{4}$ levels in $\mathrm{H}_{2} \mathrm{O}_{2}$-treated HUVECs}

As shown in Fig.4, Treatment of the cells with $\mathrm{H}_{2} \mathrm{O}_{2}$ decreased the $\mathrm{BH}_{4}$ levels in comparison with the control, whereas the decreased in $\mathrm{BH}_{4}$ level was inhibited by pre-incubation with AsIV, and there was a concentration-dependent manner. There were no difference between the control group and the AsIV H group.

\section{AsIV decreased the contents of IL-1ß, IL-6 and TNF- $\alpha$ in HUVECs treated by $\mathrm{H}_{2} \mathrm{O}_{2}$}


As shown in Fig.5, $\mathrm{H}_{2} \mathrm{O}_{2}$ treatment markedly increased the levels of IL-1 $\beta$, IL-6 and TNF- $\alpha$ in medium of HUVECs compared with the control, while pre-incubation with AsIV was demonstrated the suppressive effects on the $\mathrm{H}_{2} \mathrm{O}_{2}$-induced increase in IL- $1 \beta$, IL- 6 and TNF- $\alpha$ levels. There were no difference between the control group and the AsIV H group.

\section{Effects of AsIV on proteins expression of eNOS, iNOS, Nox4, p65 and IкB- $\alpha$ in HUVECs}

As previous reported (Hein et al. 2009; Sena et al. 2013), decrease in the ratio of eNOS dimer/monomer represents the eNOS uncoupling. The present study showed that AsIV exhibited marked suppressive effects on the decrease in the ratio of eNOS dimer/monomer induced by $\mathrm{H}_{2} \mathrm{O}_{2}$ and inhibited eNOS and Nox4 protein expression in the HUVECs. Otherwise, comparing with the control, incubation with $\mathrm{H}_{2} \mathrm{O}_{2}$ alone decreased the protein expression of p65 subunit in cytoplasm while increased the expression of p65 in nuclear fraction, increased iNOS protein expression. In addition, the cytosolic protein expression of IкB- $\alpha$ were significantly decreased in HUVECs treated by $\mathrm{H}_{2} \mathrm{O}_{2}$. These effects of $\mathrm{H}_{2} \mathrm{O}_{2}$ on p65, iNOS and I $\mathrm{kB}-\alpha$ were conversely changed by pre-incubation with AsIV, suggesting that AsIV inhibits translocation of p65 into nuclear fraction by increasing protein expression of IкB- $\alpha$ (Fig.6). There were no difference between the control group and the AsIV H group.

\section{AsIV decresed the mRNA expression of Nox4 and eNOS in HUVECs treated by $\mathrm{H}_{2} \mathrm{O}_{2}$}

Fig.7 shows $\mathrm{H}_{2} \mathrm{O}_{2}$ treatment increased mRNA expression of Nox4 and eNOS compared with the control. Pretreatment with AsIV attenuated the up-regulation of Nox4 and eNOS mRNA expression induced by $\mathrm{H}_{2} \mathrm{O}_{2}$ in a concentration-dependent manner. There were no difference between the control group and the AsIV H group. 


\section{Discussion}

It has been demonstrated that the increased level of ROS under pathological conditions is a main source of endothelial injury. Previous study has demonstrated that exposure of endothelial cells to high level $\mathrm{H}_{2} \mathrm{O}_{2}$ will induce cells to remarkable cytotoxicity (Jin et al. 2014). In our current study we demonstrated that $\mathrm{H}_{2} \mathrm{O}_{2}$ can markedly decrease the HUVECs viability. These findings were consistent with previous reports showing apoptosis in HUVECs treated by $\mathrm{H}_{2} \mathrm{O}_{2}$ (Qian et al. 2010; Wang et al. 2010). However, treatment of cells with AsIV for $1 \mathrm{~h}$ prior to exposure to $\mathrm{H}_{2} \mathrm{O}_{2}$ increased cell viability compared with the $\mathrm{H}_{2} \mathrm{O}_{2}$ alone. Otherwise, treatment with AsIV alone also increase the viability of HUVECs compared with the control group, this may be associated with that AsIV treatment could stimulate the proliferation of HUVECs (Wang et al. 2013). So we aimed to investigate the protective effects of AsIV against vascular endothelial dysfunction induced by $\mathrm{H}_{2} \mathrm{O}_{2}$ as well as the underlying mechanisms for the protection.

As is well known, NO, a key regulator of endothelial function, can inhibit apoptosis and is regarded as an endothelial cell survival factor (Wang et al. 2010). Studies have shown that the imbalance of NO and superoxide anion contributes to many cardiovascular diseases, including hypertension, atherosclerosis, and heart failure (Cave et al. 2006; Yu et al. 2016). In the previous study, it has been demonstrated that $\mathrm{H}_{2} \mathrm{O}_{2}$ activates not only eNOS but also NADPH oxidase that produce superoxide anion (Boulden et al. 2006; Cai et al. 2003a). In HUVECs, the expression level of Nox4 is 100-fold higher than that of Nox1, Nox2 or Nox5, suggesting that Nox4 is the major source of ROS (Xu et al. 2008). In normal physiological conditions, eNOS derives NO that can avidly scavenge superoxide anion and protect cells from oxidative stress-induced apoptosis (Ho et al. 2006; Tesauro et al. 2006). But uncoupling of eNOS, which occurs when levels of the eNOS cofactor 
tetrahydrobiopterin $\left(\mathrm{BH}_{4}\right)$ are suboptimal, triggers the production of superoxide instead of $\mathrm{NO}$ (Varadharaj et al. 2015). In the previous study, it has been demonstrated that subsequent $\mathrm{H}_{2} \mathrm{O}_{2}$ exposures can stimulate NADPH oxidase to derive superoxide anion. superoxide anion reacts with NO to form ONOO- which further avidly oxidizes $\mathrm{BH}_{4}$ to create uncoupled eNOS, resulting in a severe reduction of NO production (Boulden et al. 2006).

In our study, we explored the effect of AsIV on endothelial dysfunction during $\mathrm{H}_{2} \mathrm{O}_{2}$ exposure. Our results suggested that $\mathrm{H}_{2} \mathrm{O}_{2}$ significantly increased the protein and mRNA expression of Nox4 accompanied by decreased NO and increased superoxide anion production obviously. It is well known that the eNOS-derived NO reacts with superoxide anion to yield ONOO-, which can rearrange to develop more ROS, thus, leading to eNOS uncoupling and forming a viscous circle. Our current study also demonstrated that exposure to $\mathrm{H}_{2} \mathrm{O}_{2}$ can decrease cellular $\mathrm{BH}_{4}$, which is thought to be hallmarks of eNOS uncoupling. Although the eNOS protein and mRNA expression were found to be increased, the decreased protein expression of eNOS dimerization was also confirmed in HUVECs induced by $\mathrm{H}_{2} \mathrm{O}_{2}$. But pre-incubation with different concentrations of AsIV significantly inhibited $\mathrm{H}_{2} \mathrm{O}_{2}$-induced decrease in NO production with the wane of the superoxide anion production and Nox 4 protein expression, while increased eNOS dimerization expression with augment of $\mathrm{BH}_{4}$, suggesting AsIV could protect HUVECs from $\mathrm{H}_{2} \mathrm{O}_{2}$-induced injury by inhibiting eNOS uncoupling. In addition, treatment with AsIV alone could increase NO production but have no effect on the protein expression of eNOS. This may be related to the increase of the eNOS phosphorylation but not the eNOS protein expression (Wang et al. 2013).

In addition, NF- $\mathrm{NB}$ is one of the transcription factors that may be controlled by the redox status of the cell and p65 is an important subunit of NF- $\mathrm{kB}$ which involves in the transcriptional activity of 
NF- $\kappa$ B (De Martin et al. 2000; Li et al. 2006). Indeed, the endothelial generation of ROS may be a common step in all of the signaling pathways that lead to I $\kappa \mathrm{B}$ degradation and NF- $\kappa \mathrm{B}$ nuclear accumulation (Yan et al. 2015). In our current research, we found that the effect of IאB degradation and NF- $\kappa$ B transcriptional activation in HUVECs induced by $\mathrm{H}_{2} \mathrm{O}_{2}$ was inhibited by AsIV, which were consistent with the change of the Nox4 expression and the $\mathrm{O}^{2-}$ generation. The decreased levels of IL-1 $\beta$, IL-6 and TNF- $\alpha$ were detected in AsIV plus $\mathrm{H}_{2} \mathrm{O}_{2}$ groups. As is known, NF- $\kappa \mathrm{B}$ could up-regulate iNOS expression, consistent with this, the present results also showed that exposure to $\mathrm{H}_{2} \mathrm{O}_{2}$ induced increase of iNOS, while prertreatment with AsIV could inhibit the increase of iNOS protein expression. These results indicate that AsIV could play the role of protecting HUVECs treated by $\mathrm{H}_{2} \mathrm{O}_{2}$ via regulating NADPH oxidase-ROS-NF- $\kappa \mathrm{B}$ signal pathway and further reducing downstream target mRNA such as IL-1 $\beta$, IL-6 and TNF- $\alpha$.

Previous studies have been demonstrated that eNOS uncoupling mediated oxidative stress play key role in endothelium-dependent vasomotor response which is associated with the progression of myocardial hypertrophy (Davel et al. 2006). Our laboratory has been dedicated to the study of the protective effect of AsIV on cardiac hypertrophy. Our previous studies have demonstrated that oxidative stress is the critical factors contributing to apoptosis of hypertrophic cardiomyocyte and AsIV could inhibit ISO-induced apoptosis of hypertrophic cardiomyocyte via anti-oxidation (Mei et al. 2015). In current study, we have proved that AsIV also have protective effect on endothelial cells via anti-oxidation and anti-inflammatory, which can effectively inhibit the progression of myocardial hypertrophy to heart failure. In conclusion, the present studies revealed the antioxidant effect of AsIV in HUVECs. AsIV may directly inhibit Nox4 expression that produce superoxide anion stimulated by $\mathrm{H}_{2} \mathrm{O}_{2}$, suppress eNOS uncoupling and improve NO bioavailability. Moreover, AsIV may protect 
HUVECs dysfunction induced by $\mathrm{H}_{2} \mathrm{O}_{2}$ via regulating NADPH oxidase -ROS -NF- $\kappa$ B signal pathway.

Thus, the protective effect of AsIV on endothelial function may play a key role in inhibiting deterioration of myocardial hypertrophy into myocardial failure.

\section{Acknowledgments}

We thank Xiaojie Li and Jianxia Wang for their technical support. This work was supported by National Natural Science Foundation of China (81374008). The authors declare that there are no conflicts of interest associated with this work.

\section{References}

Benson, M.A., Batchelor, H., Chuaiphichai, S., Bailey, J., Zhu, H., Stuehr, D.J., et al. 2013. A pivotal role for tryptophan 447 in enzymatic coupling of human endothelial nitric oxide synthase (eNOS): effects on tetrahydrobiopterin-dependent catalysis and eNOS dimerization. J. Biol. Chem. 288: 29836-29845.

Boulden, B.M., Widder, J.D., Allen, J.C., Smith, D.A., Al-Baldawi, R.N., Harrison, D.G., et al. 2006. Early determinants of H2O2-induced endothelial dysfunction. Free Radic. Biol. Med.. 41: 810-817.

Cai, H., Griendling, K.K., and Harrison, D.G. 2003a. The vascular NAD(P)H oxidases as therapeutic targets in cardiovascular diseases. Trends Pharmacol. Sci. 24: 471-478.

Cai, H., Li, Z., Davis, M.E., Kanner, W., Harrison, D.G., Dudley, S.C., et al. 2003b. Akt-dependent phosphorylation of serine 1179 and mitogen-activated protein kinase kinase/extracellular signal-regulated kinase 1/2 cooperatively mediate activation of the endothelial nitric-oxide synthase by hydrogen peroxide. Mol. Pharmacol. 63: 325-331.

Cai, S., Khoo, J., and Channon, K.M. 2005. Augmented BH4 by gene transfer restores nitric oxide synthase function in hyperglycemic human endothelial cells. Cardiovasc. Res. 65: 823-831. 
Cave, A.C., Brewer, A.C., Narayanapanicker, A., Ray, R., Grieve, D.J., Walker, S., et al. 2006. NADPH oxidases in cardiovascular health and disease. Antioxid. Redox Signaling, 8: 691-728.

Chen, J., and Mehta, J.L. 2004. Role of oxidative stress in coronary heart disease. Indian Heart J. 56: $163-173$

Davel, A.P., Brum, P.C., and Rossoni, L.V. 2014. Isoproterenol induces vascular oxidative stress and endothelial dysfunction via a Gialpha-coupled beta2-adrenoceptor signaling pathway. PloS one, 9: e91877.

Davel, A.P., Kawamoto, E.M., Scavone, C., Vassallo, D.V., and Rossoni, L.V. 2006. Changes in vascular reactivity following administration of isoproterenol for 1 week: a role for endothelial modulation. Br. J. Pharmacol. 148: 629-639.

De Martin, R., Hoeth, M., Hofer-Warbinek, R., and Schmid, J.A. 2000. The transcription factor NF-kappa B and the regulation of vascular cell function. Arterioscler. Thromb. Vasc. Biol. 20: E83-88.

Drummond, G.R., Cai, H., Davis, M.E., Ramasamy, S., and Harrison, D.G. 2000. Transcriptional and posttranscriptional regulation of endothelial nitric oxide synthase expression by hydrogen peroxide. Circ. Res. 86: 347-354.

Forstermann, U. 2010. Nitric oxide and oxidative stress in vascular disease. Pflugers Arch. 459: 923-939.

Forstermann, U., and Munzel, T. 2006. Endothelial nitric oxide synthase in vascular disease: from marvel to menace. Circulation. 113: 1708-1714.

Gorren, A.C., and Mayer, B. 2002. Tetrahydrobiopterin in nitric oxide synthesis: a novel biological role for pteridines. Curr. Drug Metab. 3: 133-157.

Hein, T.W., Singh, U., Vasquez-Vivar, J., Devaraj, S., Kuo, L., and Jialal, I. 2009. Human C-reactive 
protein induces endothelial dysfunction and uncoupling of eNOS in vivo. Atherosclerosis. 206: 61-68. Hermida, N., and Balligand, J.L. 2014. Low-density lipoprotein-cholesterol-induced endothelial dysfunction and oxidative stress: the role of statins. Antioxid. Redox Signaling, 20: 1216-1237.

Ho, F.M., Lin, W.W., Chen, B.C., Chao, C.M., Yang, C.R., Lin, L.Y., et al. 2006. High glucose-induced apoptosis in human vascular endothelial cells is mediated through NF-kappaB and c-Jun NH2-terminal kinase pathway and prevented by PI3K/Akt/eNOS pathway. Cell. Signalling, 18: 391-399.

Jaimes, E.A., Sweeney, C., and Raij, L. 2001. Effects of the reactive oxygen species hydrogen peroxide and hypochlorite on endothelial nitric oxide production. Hypertension. 38: 877-883.

Jin, Y., Liu, K., Peng, J., Wang, C., Kang, L., Chang, N., et al. 2014. Rhizoma Dioscoreae Nipponicae polysaccharides protect HUVECs from HO-induced injury by regulating PPARgamma factor and the NADPH oxidase/ROS-NF-kappaB signal pathway. Toxicol. Lett. 232: 149-158.

Kang, G.J., Han, S.C., Yoon, W.J., Koh, Y.S., Hyun, J.W., Kang, H.K., et al. 2013. Sargaquinoic acid isolated from Sargassum siliquastrum inhibits lipopolysaccharide-induced nitric oxide production in macrophages via modulation of nuclear factor-kappaB and c-Jun N-terminal kinase pathways. Immunopharmacol. Immunotoxicol. 35: 80-87.

Li, Q., Harraz, M.M., Zhou, W., Zhang, L.N., Ding, W., Zhang, Y., et al. 2006. Nox2 and Rac1 regulate H2O2-dependent recruitment of TRAF6 to endosomal interleukin-1 receptor complexes. Mol. Cell. Biol. 26: 140-154.

Lu, M., Tang, F., Zhang, J., Luan, A., Mei, M., Xu, C., et al. 2015. Astragaloside IV attenuates injury caused by myocardial ischemia/reperfusion in rats via regulation of toll-like receptor $4 /$ nuclear factor-kappaB signaling pathway. Phytother. Res. 29: 599-606.

Ma, Y., Li, W., Yin, Y., and Li, W. 2015. AST IV inhibits $\mathrm{H}_{2} \mathrm{O}_{2}$-induced human umbilical vein 
endothelial cell apoptosis by suppressing Nox4 expression through the TGF-beta1/Smad2 pathway. Int. J. Mol. Med. 35: 1667-1674.

Makarenko, V.V., Usatyuk, P.V., Yuan, G., Lee, M.M., Nanduri, J., Natarajan, V., et al. 2014. Intermittent hypoxia-induced endothelial barrier dysfunction requires ROS-dependent MAP kinase activation. Am. J. Physiol. 306: C745-752.

Mei, M., Tang, F., Lu, M., He, X., Wang, H., Hou, X., et al. 2015. Astragaloside IV attenuates apoptosis of hypertrophic cardiomyocyte through inhibiting oxidative stress and calpain-1 activation. Environ. Toxicol. Pharmacol. 40: 764-773.

Moens, A.L., and Kass, D.A. 2006. Tetrahydrobiopterin and cardiovascular disease. Arterioscler. Thromb. Vasc. Biol. 26: 2439-2444.

Napoli, C., Williams-Ignarro, S., De Nigris, F., Lerman, L.O., Rossi, L., Guarino, C., et al. 2004. Long-term combined beneficial effects of physical training and metabolic treatment on atherosclerosis in hypercholesterolemic mice. Proc. Natl. Acad. Sci. U.S.A. 101: 8797-8802.

Olejarz, W., Bryk, D., Zapolska-Downar, D., Malecki, M., Stachurska, A., and Sitkiewicz, D. 2014. Mycophenolic acid attenuates the tumour necrosis factor-alpha-mediated proinflammatory response in endothelial cells by blocking the MAPK/NF-kappaB and ROS pathways. Eur. J. Clin. Invest. 44: 54-64.

Qian, J., Jiang, F., Wang, B., Yu, Y., Zhang, X., Yin, Z., et al. 2010. Ophiopogonin D prevents H2O2-induced injury in primary human umbilical vein endothelial cells. J. Ethnopharmacol. 128: 438-445.

Qiu, L.H., Xie, X.J., and Zhang, B.Q. 2010. Astragaloside IV improves homocysteine-induced acute phase endothelial dysfunction via antioxidation. Biol. Pharm. Bull. 33: 641-646. 
Sena, C.M., Pereira, A.M., and Seica, R. 2013. Endothelial dysfunction - a major mediator of diabetic vascular disease. Biochim. Biophys. Acta, 1832: 2216-2231.

Tesauro, M., Thompson, W.C., and Moss, J. 2006. Effect of staurosporine-induced apoptosis on endothelial nitric oxide synthase in transfected COS-7 cells and primary endothelial cells. Cell Death Differ. 13: 597-606.

Varadharaj, S., Porter, K., Pleister, A., Wannemacher, J., Sow, A., Jarjoura, D., et al. 2015. Endothelial nitric oxide synthase uncoupling: a novel pathway in OSA induced vascular endothelial dysfunction. Respir. Physiol. Neurobiol. 207: 40-47.

Vendrov, A.E., Vendrov, K.C., Smith, A., Yuan, J., Sumida, A., Robidoux, J., et al. 2015. NOX4 NADPH Oxidase-Dependent Mitochondrial Oxidative Stress in Aging-Associated Cardiovascular Disease. Antioxid. Redox Signaling, 23(18):1389-409.

Wang, S.G., Xu, Y., Chen, J.D., Yang, C.H., and Chen, X.H. 2013. Astragaloside IV stimulates angiogenesis and increases nitric oxide accumulation via JAK2/STAT3 and ERK1/2 pathway. Molecules (Basel, Switzerland). 18: 12809-12819.

Wang, S.L., Liu, D.S., Liang, E.S., Gao, Y.H., Cui, Y., Liu, Y.Z., et al. 2015. Protective effect of allicin on high glucose/hypoxia-induced aortic endothelial cells via reduction of oxidative stress. Exp. Ther. Med. 10: 1394-1400.

Wang, Y.K., Hong, Y.J., Wei, M., Wu, Y., Huang, Z.Q., Chen, R.Z., et al. 2010. Curculigoside attenuates human umbilical vein endothelial cell injury induced by H2O2. J. Ethnopharmacol. 132: 233-239.

Xu, H., Goettsch, C., Xia, N., Horke, S., Morawietz, H., Forstermann, U., et al. 2008. Differential roles of PKCalpha and PKCepsilon in controlling the gene expression of Nox4 in human endothelial cells. 
Free Radic. Biol. Med. 44: 1656-1667.

Yan, S., Zhang, X., Zheng, H., Hu, D., Zhang, Y., Guan, Q., et al. 2015. Clematichinenoside inhibits VCAM-1 and ICAM-1 expression in TNF-alpha-treated endothelial cells via NADPH oxidase-dependent IkappaB kinase/NF-kappaB pathway. Free Radic. Biol. Med. 78: 190-201.

Yang, J., Wang, H.X., Zhang, Y.J., Yang, Y.H., Lu, M.L., Zhang, J., et al. 2013. Astragaloside IV attenuates inflammatory cytokines by inhibiting TLR4/NF- $\mathrm{\kappa B}$ signaling pathway in isoproterenol-induced myocardial hypertrophy. J. Ethnopharmacol. 150(3):1062-70.

Yu, Y., Xiong, Y., Montani, J.P., Yang, Z., and Ming, X.F. 2016. En Face Detection of Nitric Oxide and Superoxide in Endothelial Layer of Intact Arteries. J. Vis. Exp. (108): e53718.

Zhang, S., Tang, F., Yang, Y., Lu, M., Luan, A., Zhang, J., et al. 2015. Astragaloside IV protects against isoproterenol-induced cardiac hypertrophy by regulating NF-kappaB/PGC-1alpha signaling mediated energy biosynthesis. PloS one, 10: e0118759.

Zhang, W.J., Hufnagl, P., Binder, B.R., and Wojta, J. 2003. Antiinflammatory activity of astragaloside IV is mediated by inhibition of NF-kappaB activation and adhesion molecule expression. Thromb. Haemostasis, 90: 904-914.

Zhao, Q.D., Viswanadhapalli, S., Williams, P., Shi, Q., Tan, C., Yi, X., et al. 2015. NADPH oxidase 4 induces cardiac fibrosis and hypertrophy through activating Akt/mTOR and NFkappaB signaling pathways. Circulation. 131: 643-655. 


\section{Legends for figures}

Fig.1. Cell viability of HUVECs assayed by MTT. The results are expressed as percentage of control. Values are presented as the means \pm SEM from six independent experiments. ${ }^{*}: P<0.05$ is considered statistical significance.

Fig.2. Effects of AsIV on NO levels in the medium of HUVECs. Values are presented as the means \pm SEM from six independent experiments. ${ }^{*}: P<0.05$ is considered statistical significance.

Fig.3. Effects of AsIV on superoxide anion generation in HUVECs. a: representative fluorographs of intracellular superoxide anion. b: the superoxide anion production measured by microplate reader. Values are presented as the means \pm SEM from six independent experiments. ${ }^{*}: P<0.05$ is considered statistical significance.

Fig.4. AsIV increased $\mathrm{BH}_{4}$ levels in $\mathrm{H}_{2} \mathrm{O}_{2}$-treated HUVECs. Values are presented as the means \pm SEM from four independent experiments. $*$ : $P<0.05$ is considered statistical significance.

Fig.5. Effects of AsIV on the levels of IL-1 $\beta$ and IL-6 and TNF- $\alpha$ in the medium of HUVECs. a: The levels of IL-1 $\beta$. b: The levels of IL-6. c: The levels of TNF- $\alpha$. Values are presented as the means \pm SEM from six independent experiments. $*: P<0.05$ is considered statistical significance.

Fig.6. Effects of AsIV on proteins expression of eNOS, iNOS, Nox4, p65 and IкB- $\alpha$ in HUVECs measured by western blot. a: representatives of western graph of eNOS expression (upper panel) and its statistical data (lower panel). b: representatives of western graph of eNOS dimer, monomer respectively (upper panel) and the statistical data of the ratio of dimer : monomer (lower panel), which represents protein dimerization in HUVECs. c: representatives of western graph of iNOS iexpression (upper panel) and its statistical data (lower panel). d: representatives of western graph of Nox4 expression (upper panel) and its statistical data (lower panel). e: representatives of western graph of 
p65 expression in nucleus (upper panel) and its statistical data (lower panel). f: representatives of western graph of p65 expression in cytoplasm (upper panel) and its statistical data (lower panel). g: representatives of western graph of I $\mathrm{I} B-\alpha$ expression (upper panel) and its statistical data (lower panel).Values are presented as the means \pm SEM from six independent experiments. *: $P<0.05$ is considered statistical significance.

Fig.7. Effects of AsIV on mRNA expression of Nox4 and eNOS in HUVECs measured by RT-PCR. a: representatives of RT-PCR graph of eNOS (upper panel) and its statistical data (lower panel).b: representatives of RT-PCR graph of Nox4 (upper panel) and its statistical data (lower panel). Values are presented as the means \pm SEM from six independent experiments. *: $P<0.05$ is considered statistical significance. 


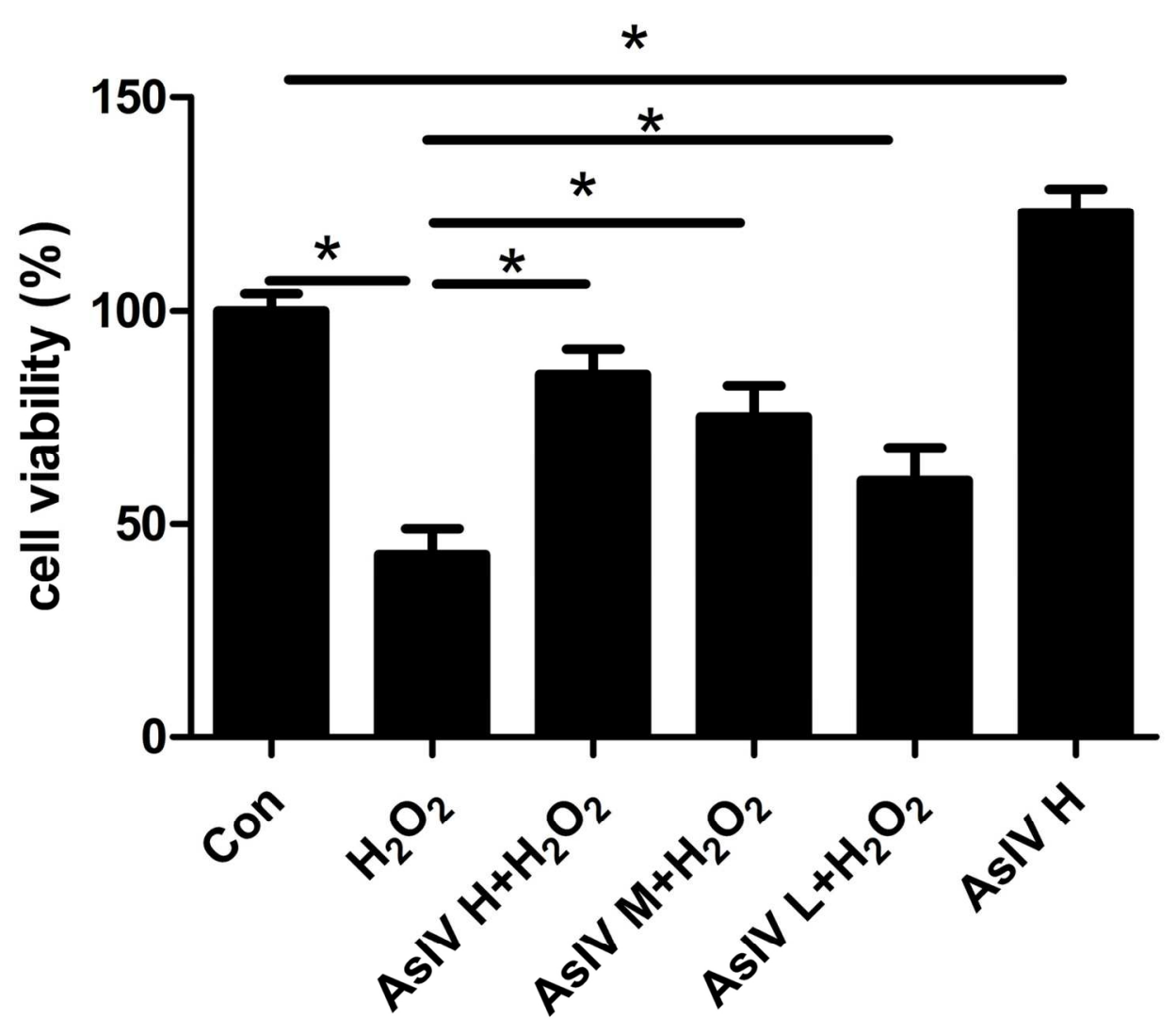

$67 \times 60 \mathrm{~mm}(600 \times 600$ DPI $)$ 


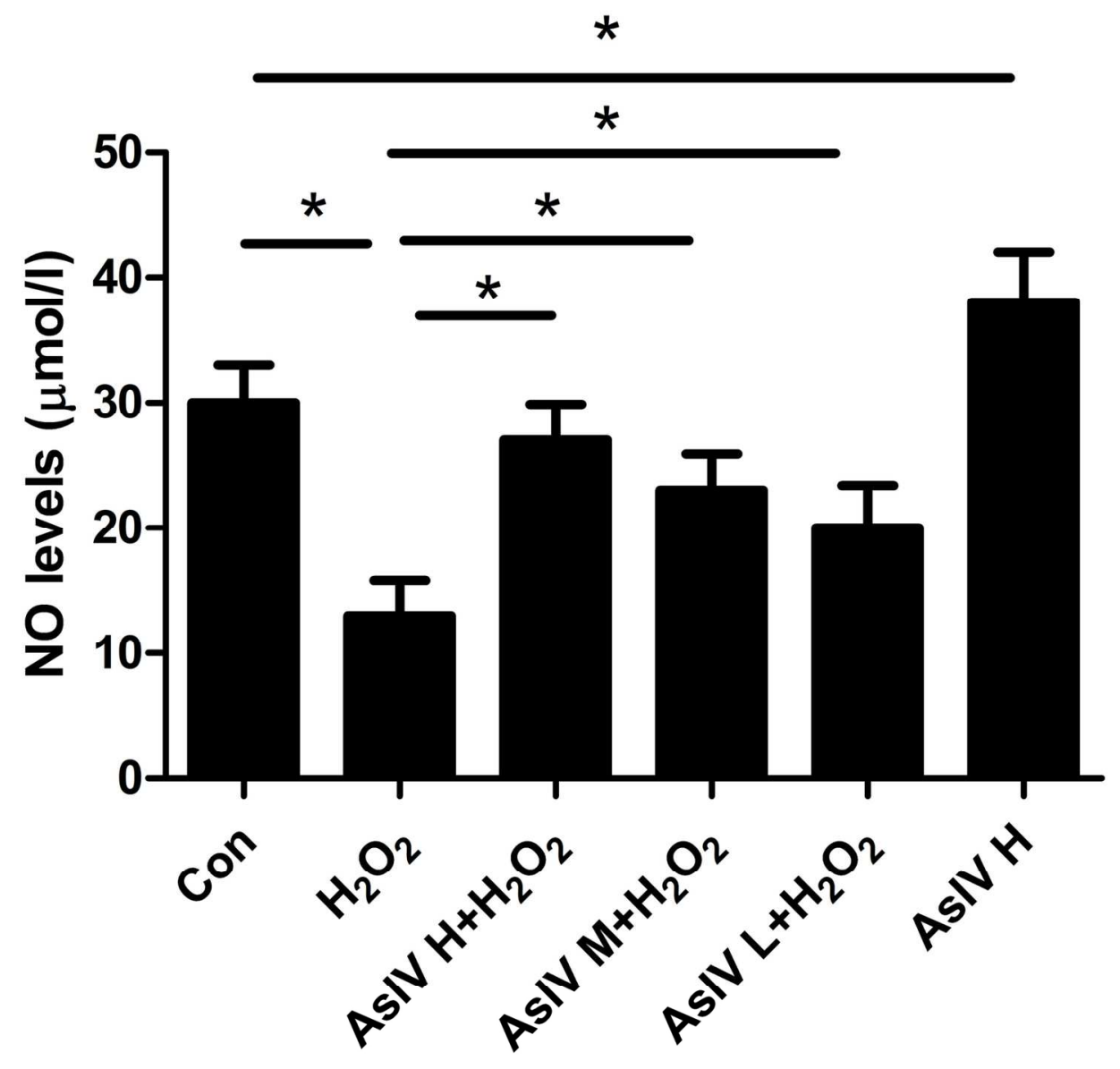

$73 \times 71 \mathrm{~mm}(600 \times 600 \mathrm{DPI})$ 


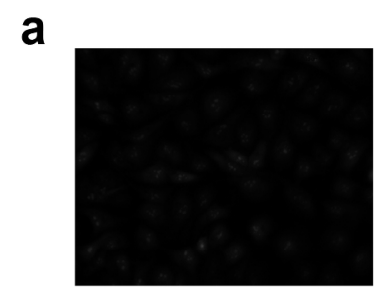

Con

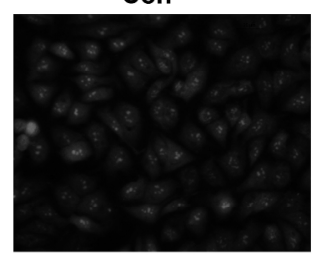

AsIV $\mathrm{M}+\mathrm{H}_{2} \mathrm{O}_{2}$

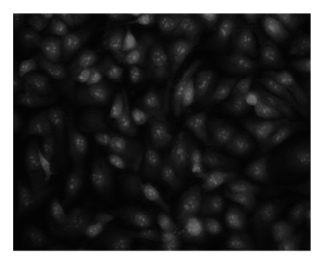

$\mathrm{H}_{2} \mathrm{O}_{2}$

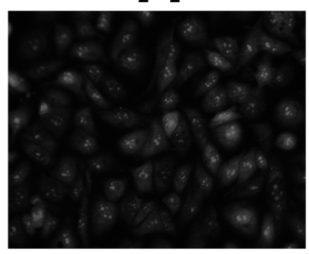

b

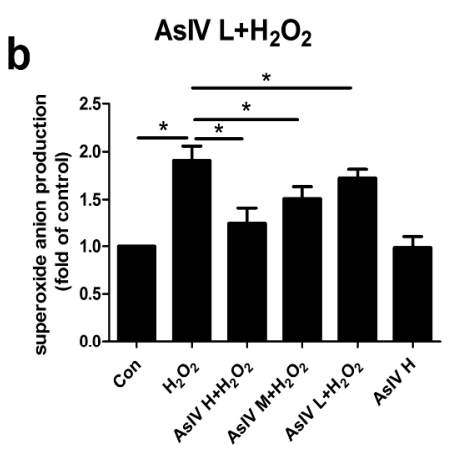

$150 \times 125 \mathrm{~mm}(600 \times 600 \mathrm{DPI})$

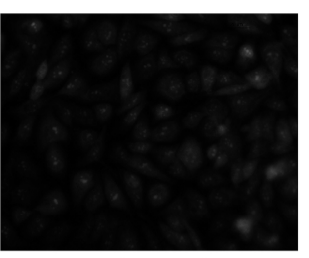

AsIV $\mathrm{H}+\mathrm{H}_{2} \mathrm{O}_{2}$

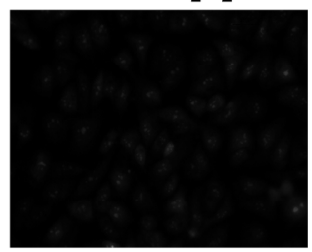

AsIV H 


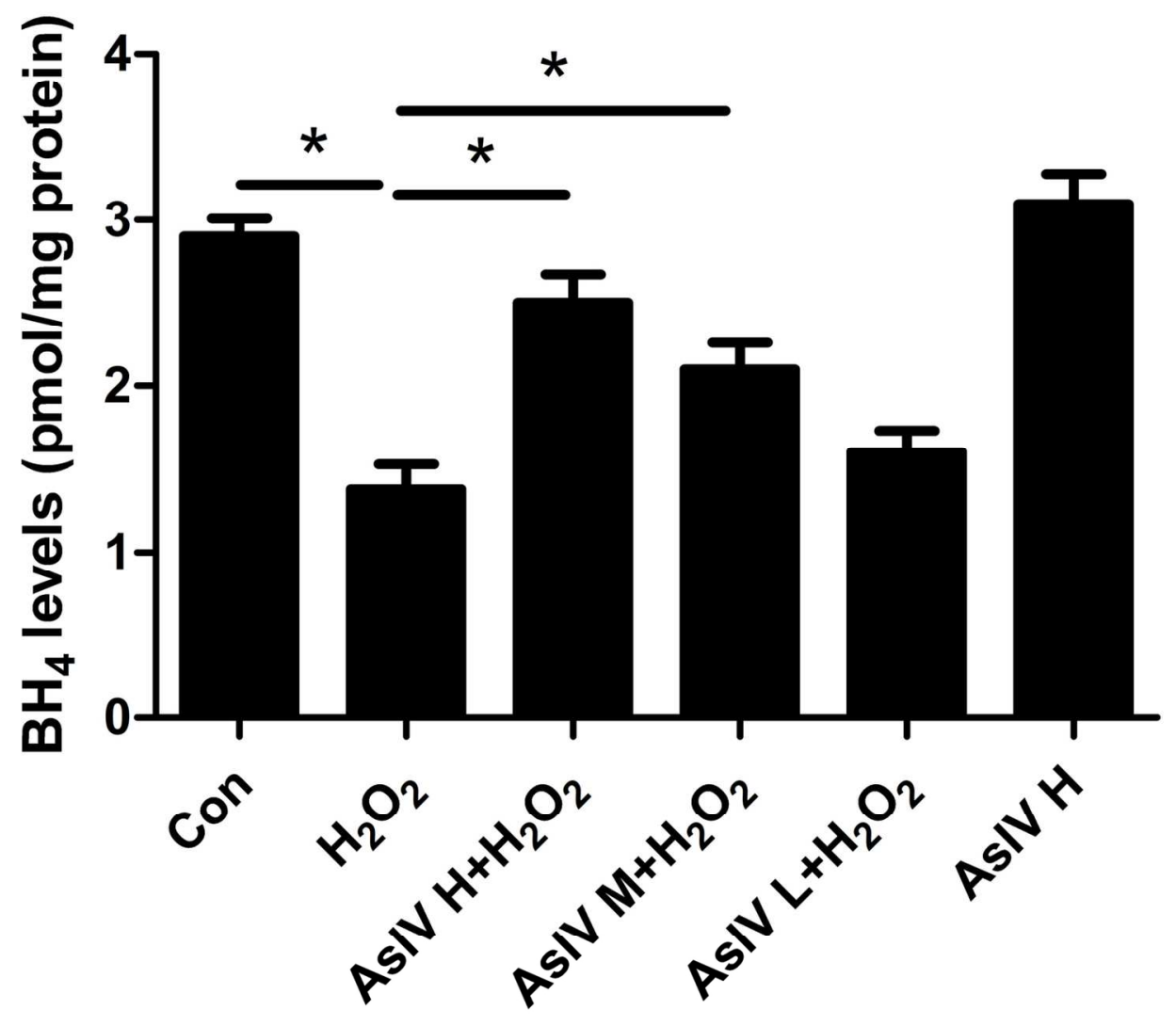

$67 \times 59 \mathrm{~mm}(600 \times 600 \mathrm{DPI})$ 

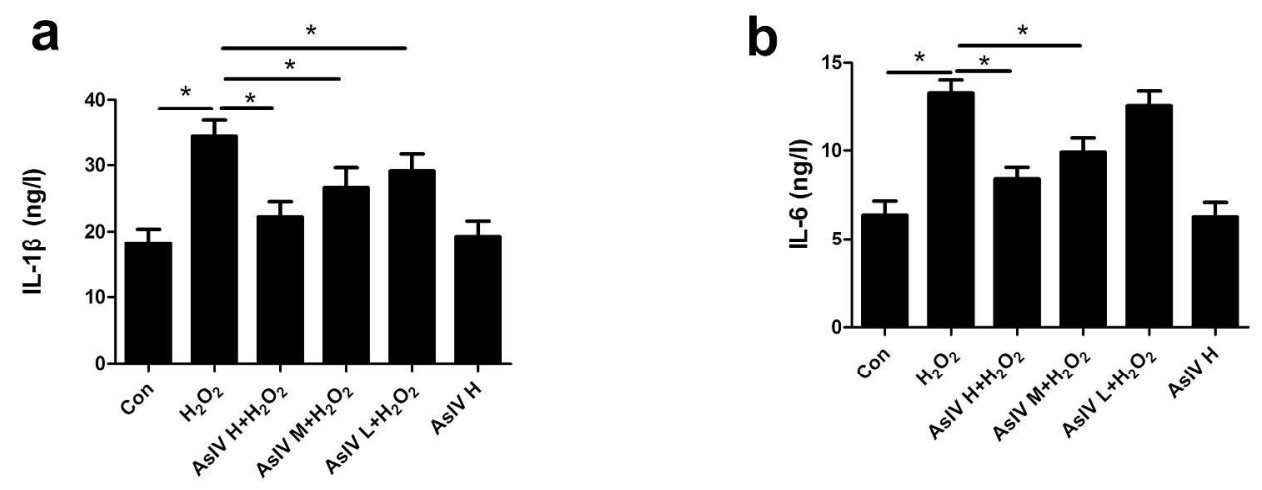

C

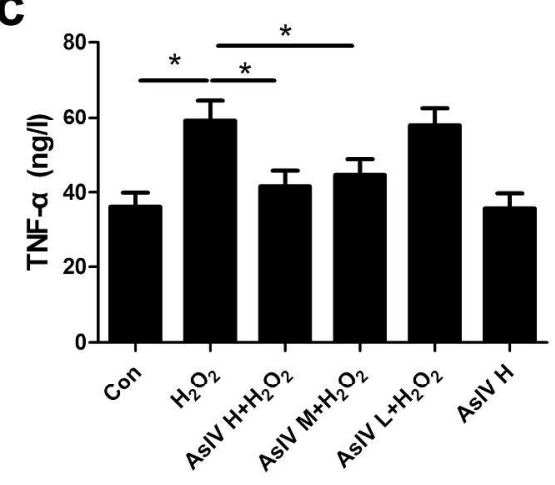

$141 \times 115 \mathrm{~mm}(600 \times 600 \mathrm{DPI})$ 

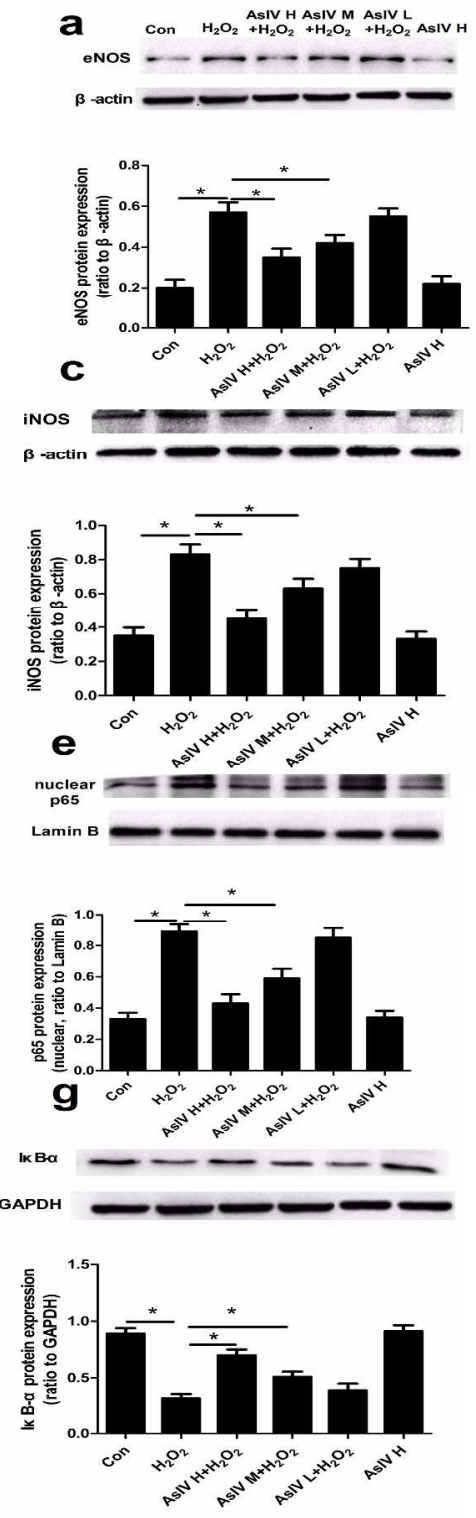
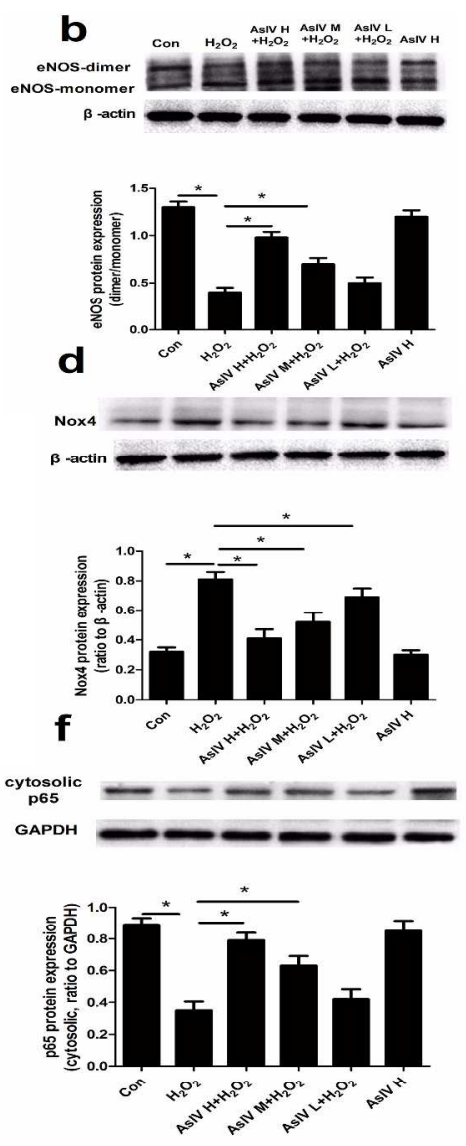

$229 \times 311 \mathrm{~mm}(600 \times 600 \mathrm{DPI})$ 

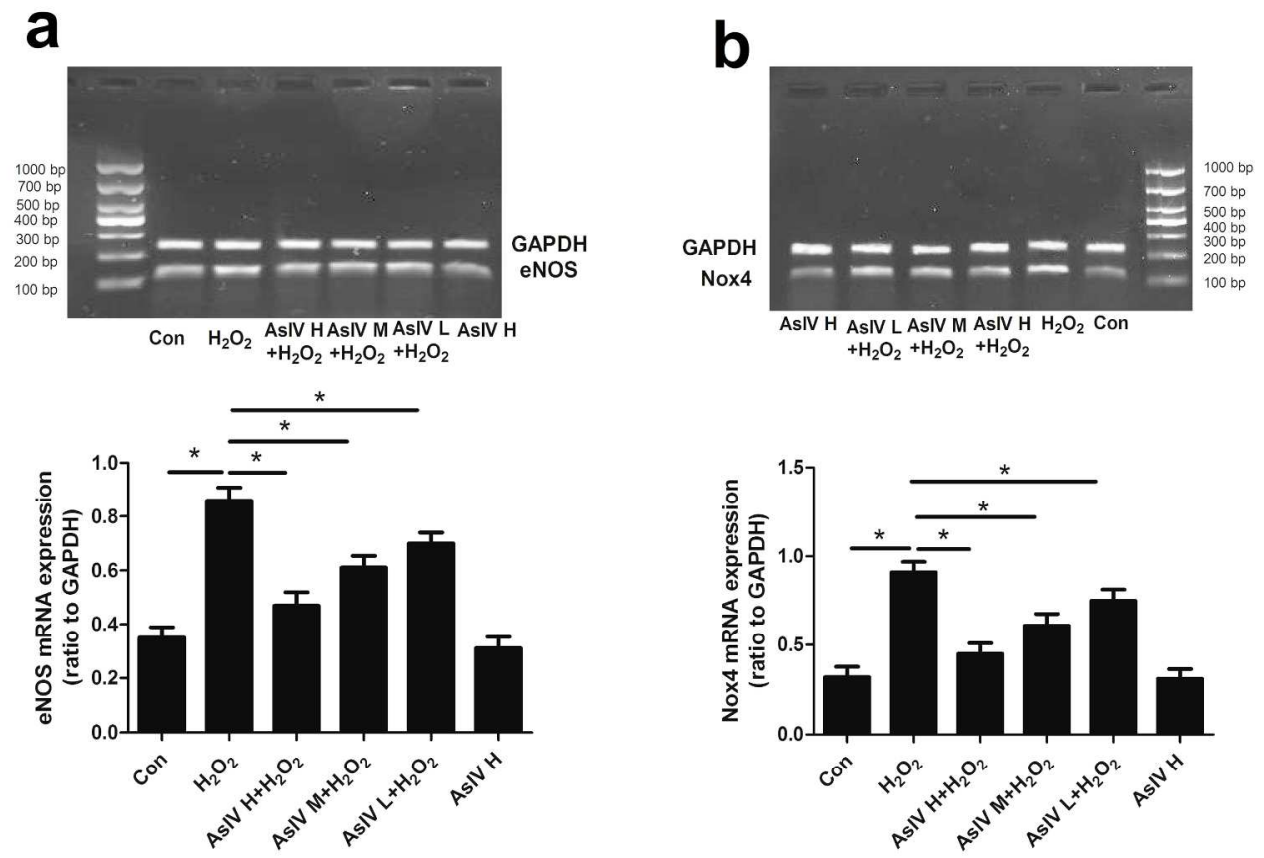

$118 \times 85 \mathrm{~mm}(600 \times 600$ DPI $)$ 